\title{
9. CONCLUSIONS
}

(1) Although the input motion of cam followers is periodical, transient responses characterise their vibrations (sec. 1.3).

(2) The lowest natural frequency of a mechanism largely dominates the transient response (sec. 3.7.3).

(3) Flexibility of the driving members is one of the most important factors in cam follower vibrations (secs 4.5.4, 4.7, 5.5.3 and 7.6).

(4) In practice the flywheel effect of the cam is negligible, and it should be kept so (secs 5.5.5 and 5.7).

(5) The 4-degrees-of-freedom model DYNACAM permits of detailed analysis of machine dynamics by means of simulation. Even nonlinear phenomena such as backlash, squeeze and impact can be simulated (sec. 7.4.3).

(6) The single-degree-of-freedom model CAMSHAFT-1 proves to be an adequate tool for predicting the amplitude of the residual vibration of a cam follower driven by a relatively flexible shaft (sec. 8.2.5), despite the fact that considerable simplifications have been introduced by the assumptions that the stiffness is a function of the nominal follower velocity and that the input motion is equal to the nominal follower displacement (secs 4.3.1 and 5.5.5).

(7) Residual-vibration amplitudes can be reduced by the introduction of cam curves with moderate maximum nominal velocity. The same improvement, however, can be achieved by a slight increase of the shaft diameter; hence there are no compelling reasons to abolish the well-known cycloidal as a basic cam curve (sec. 8.4).

(8) Rayleigh's method, and the assumption that the lowest mode of vibration is equal to the static deflection, together form a practical way, with satisfactory accuracy, for calculating the lowest natural frequency (sec. 4.6 and appendix 3).

(9) The rules of design based on the CAMSHAFT-1 model (sec. 8.5) and the simple method for calculation of the lowest natural frequency (sec. 4.6) constitute a method for designing machines which satisfy requirements concerning positional accuracy and dynamic load, while laborious calculations can be avoided. 